\title{
C-Glycosylflavonoids
}

\section{THE CHEMISTRY OF ORIENTIN AND ISO-ORIENTIN}

\author{
BY B. H. KOEPPEN \\ Department of Food Science, University of Stellenbosch, Stellenbosch \\ AND D. G. ROUX \\ Leather Industries Research Institute, Rhodes University, Grahamstown, South Africa
}

(Received 22 March 1965)

\begin{abstract}
1. The structures of orientin and iso-orientin have been investigated by periodic acid and ferric chloride oxidation of the tetra- $O$-methyl derivatives and by identification of the products of oxidation. 2. The preparation and properties of the $3^{\prime}, 4^{\prime}, 7$ trimethyl ethers of orientin and iso-orientin aredescribed, and thenuclear-magneticresonance spectra of orientin, iso-orientin and their tri- and tetra-O-methyl derivatives are discussed. 3. Orientin and iso-orientin are formulated as 8- and 6- $C$ - $\beta$-D-glucopyranosyl-luteolin respectively.
\end{abstract}

In a previous communication (Koeppen, 1962) evidence was presented that orientin and homoorientin are isomeric $C$-glycosyl-luteolin compounds. In view of this Professor L. J. Haynes (personal communication) has suggested that homo-orientin be renamed iso-orientin, and it is intended to follow this suggestion in the present and in all future references to this compound. Subsequently Koeppen (1964) claimed that both orientin and isoorientin (homo-orientin) possess $C$-glucopyranosyl residues, their interconvertibility in hot dilute aqueous acid media being due to a WesselyMoser rearrangement of the flavone portion of the molecule.

The present paper provides details of the experimental methods that led to the above conclusions, and a description is given of the preparation and properties of derivatives that have provided valuable information on the structural interrelationship of the parent compounds. Nuclear-magneticresonance (n.m.r.) evidence is also presented that favours the identification of orientin as 8-C- $\beta-\mathrm{D}$ glucopyranosyl-luteolin (I) and iso-orientin as the corresponding 6-C- $\beta$-D-glucopyranosylflavone (II).

\section{EXPERIMENTAL AND RESULTS}

Infrared-absorption spectra were recorded on a PerkinElmer model 21 spectrometer by the KBr-disk method $(0.3-0.6 \mathrm{mg}$. of compound $/ 250 \mathrm{mg}$. of $\mathrm{KBr})$. C, H, methoxyl and acetyl determinations were by $K$. Jones, Microanalytical Laboratory, C.S.I.R., Pretoria, South Africa. All melting points are uncorrected and were determined by the Kofler method. Mixed melting points were determined by the method of Roux \& Maihs (1960).

\section{Oxidations of $3^{\prime}, 4^{\prime}, 5,7-t e t r a-0-m e t h y l o r i e n t i n$ and $3^{\prime}-4^{\prime}, 5,7$-tetra-0-methyliso-orientin}

The tetramethyl ethers were prepared as described by Koeppen, Smit \& Roux (1962), recrystallized to constant m.p. and dried for $16 \mathrm{hr}$. over $\mathrm{P}_{2} \mathrm{O}_{5}$ at $110^{\circ}$ under reduced pressure.

Periodic acid oxidation. The method employed was essentially the Fleury-Lange procedure described by Dyer (1956). The compound (14.0 mg.) in aq. $60 \%(v / v)$ ethanol $\left(20 \mathrm{ml}\right.$.) was treated with aq. $0 \cdot 04 \mathrm{~N}-\mathrm{HIO}_{4}(20 \mathrm{ml}$.) in the dark at $15^{\circ}$. A blank was similarly prepared. The rate of oxidation was followed by withdrawing samples $(4 \mathrm{ml}$.) of the reaction mixture and the blank at various intervals and adding to each saturated aq. $\mathrm{NaHCO}_{3}$ soln. (10 ml.), standard $0.02 \mathrm{~N}-\mathrm{NaAsO}_{2}$ soln. (5ml.) and $20 \%(\mathrm{w} / \mathrm{v}) \mathrm{KI}$ in saturated aq. $\mathrm{NaHCO}_{3}$ soln. (1ml.). After standing (15 min.) the mixtures were titrated with standard $0.02 \mathrm{~N}-\mathrm{I}_{2}$ soln. and starch indicator.

Although the initial uptake of periodate was faster for $3^{\prime}, 4^{\prime}, 5,7$-tetra- $O$-methyliso-orientin than for $3^{\prime}, 4^{\prime}, 5,7$-tetra$O$-methylorientin, each compound consumed only $2.0 \mathrm{~mol}$./ mol. after $23 \mathrm{hr}$. (Koeppen, 1962). No additional uptake of periodate was observed during a further $24 \mathrm{hr}$. Control experiments with methyl $\alpha$-D-glucopyranoside and methyl $\alpha$-D-galactopyranoside under the above conditions also resulted in the uptake of only $2.0 \mathrm{~mol}$. of periodate $/ \mathrm{mol}$. of compound. This was attributed to the expected cleavage of the $\alpha$-glycol groupings only, as no hydrolysis of the methyl glycosides could be detected after $48 \mathrm{hr}$. in aq. $\mathrm{HCl}$ at the same temperature and $\mathrm{pH}(2 \cdot 2)$ as the $\mathrm{HIO}_{4}$ solutions.

Formic acid determination (cf. Dyer, 1956). The compound $(50.0 \mathrm{mg}$.$) in aq. 60 \%(\mathrm{v} / \mathrm{v})$ ethanol $(75 \mathrm{ml}$.) was treated in the dark with aq. $0.04 \mathrm{~N}-\mathrm{HIO}_{4}\left(75 \mathrm{ml}\right.$.) for $43 \mathrm{hr}$. at $15^{\circ}$. Aq. $5 \cdot 6 \%(w / v) ~ B a(O H)_{2}$ soln. ( $8 \mathrm{ml}$.) was added and the precipitate was removed by centrifugation after lhr. at $0^{\circ}$. The precipitate was washed with several portions of water and the combined supernatant and washings (about 
$220 \mathrm{ml}$.) were evaporated to dryness under reduced pressure at $60^{\circ}$. The residue was dissolved in water $(10 \mathrm{ml}$.), acidified to litmus by the dropwise addition of $10 \%(w / v) \mathrm{H}_{3} \mathrm{PO}_{4}$ and distilled to dryness under reduced pressure at $70^{\circ}$, the acid distillate being collected in an ice-cooled receiver. The non-volatile residue was dissolved in water $(10 \mathrm{ml}$.) and distilled to dryness three times as above. The four combined acid distillates were titrated to $\mathrm{pH} 7 \cdot 0$ with standard NaOH. A blank determination was made similarly. The acidity, expressed as formic acid, revealed that 0.8 and $0.9 \mathrm{~mol}$. of acid were produced $/ \mathrm{mol}$. of $3^{\prime}, 4^{\prime}, 5,7$-tetra- $O$ methylorientin and $3^{\prime}, 4^{\prime}, 5,7$-tetra- $O$-methyliso-orientin respectively. The neutralized distillate was evaporated to a small volume (about $10 \mathrm{ml}$.) under reduced pressure at $70^{\circ}$ and treated with aq. $10 \%(\mathrm{w} / \mathrm{v}) \mathrm{HgCl}_{2}(2 \mathrm{ml}$.), saturated aq. sodium acetate soln. ( $1 \mathrm{ml}$.) and aq. $4 \mathrm{~N}-\mathrm{HCl}(0.2 \mathrm{ml}$.). The mixture was immersed in boiling water in the dark for $1 \mathrm{hr}$. The calomel formed was equivalent to $0.8 \mathrm{~mol}$. of formic acid/mol. of $3^{\prime}, 4^{\prime}, 5,7$-tetra-O-methylorientin. An identical result was obtained for $3^{\prime}, 4^{\prime}, 5,7$-tetra-O-methyliso-orientin.

Glycerol detection. Glycerol was detected as a product of $\mathrm{HIO}_{4}$ oxidation, $\mathrm{NaBH}_{4}$ reduction and acid hydrolysis of $3^{\prime}, 4^{\prime}, 5,7$-tetra-O-methylorientin and $3^{\prime}, 4^{\prime}, 5,7$-tetra- $O$ methyliso-orientin by the procedure described by Koeppen (1965a).

Ferric chloride oxidation. The method of Hay \& Haynes (1956) was considerably modified for use on a semi-micro scale. The tetramethyl ether (about $25 \mathrm{mg}$.) and $\mathrm{FeCl}_{3}$ $(0.2 \mathrm{~g}$.) in water $(0.8 \mathrm{ml}$.) were refluxed gently for $6 \mathrm{hr}$. The mixture was cooled, the $\mathrm{pH}$ adjusted to 8.0 with aq. $\mathrm{NaOH}$ soln. and the precipitate was removed by centrifugation. The $\mathrm{pH}$ of the supernatant was adjusted to 7.0 with aq. $\mathrm{HCl}$ and the solution was desalted in a Shandon electrolytic desalting apparatus (Mark II). The salt-free solution was concentrated to a small volume (about $0.5 \mathrm{ml}$.) and examined by paper chromatography. Both $3^{\prime}, 4^{\prime}, 5,7$-tetra-O-methylorientin and $3^{\prime}, 4^{\prime}, 5,7$-tetra- $O$-methyliso-orientin yielded a mixture of glucose and arabinose in about equal proportions, indistinguishable from authentic $\mathrm{D}$-glucose and $\mathrm{D}$-arabinose respectively by co-chromatography in benzene-butan-1-olpyridine-water (1:5:3:3, by vol.), in butan-1-ol-acetic acid-water $(20: 5: 11$, by vol.) and in aq. $75 \%(w / w)$ phenol. Oxidation of the parent compounds, orientin and isoorientin, under the above conditions yielded similar results, but with barbaloin (isolated from commercial aloin) arabinose was the major product and only a trace of glucose could be detected (cf. Hay \& Haynes, 1956).

\section{Orientin and iso-orientin trimethyl ethers}

3',4',7-Tri-0-methylorientin. The most satisfactory method of preparation was found to be by methylation of orientin with dimethyl sulphate as follows. Orientin (130 mg.) was refluxed with dimethyl sulphate (0.3g.) and anhydrous $\mathrm{K}_{2} \mathrm{CO}_{3}(0.8 \mathrm{~g}$.) in acetone $(15 \mathrm{ml}$.) for $3 \mathrm{hr}$. The solution was filtered and the residue was washed with three small portions of cold acetone. The filtrate and washings were combined, concentrated under reduced pressure and applied as a band along the short side of two Whatman no. 3 paper sheets $(46 \mathrm{~cm} . \times 57 \mathrm{~cm}$.). The acetone-insoluble residue was dissolved in water $(40 \mathrm{ml}$.) and acidified with aq. HCl. The precipitated orientin (about $60 \mathrm{mg}$.) was recovered by filtration after $24 \mathrm{hr}$. at about $5^{\circ}$, washed until free from acid and dried. The methylation procedure was repeated four times as described. The ten Whatman no. 3 paper sheets containing the acetone-soluble methylation products were developed by downward migration in butan-1-ol-acetic acid-water (20:5:11, by vol.). Examination of the dried chromatograms under u.v. light (3650 $\AA$ ) revealed a dark band $\left(R_{F}\right.$ about 0.45$)$ as the major component. The band was cut out and eluted with aq. $70 \%$ ( $/ / v)$ ethanol. Complete elution of the material took several days. The eluate was evaporated to dryness under reduced pressure and the residue was recrystallized to constant m.p. from water. After drying to constant weight under reduced pressure at $110^{\circ}$, the yield was $138 \mathrm{mg}$. of pale-yellow prisms, m.p. $224^{\circ}$, solidifying on further heating and remelting at $271-273^{\circ} ;[\alpha]_{D}^{24}-37 \cdot 7 \pm 0.4^{\circ}$ (c 1.85 in pyridine); $\lambda_{\max } .252$, 272 and $348 \mathrm{~m} \mu$ in ethanol (log $\epsilon 4 \cdot 11,4 \cdot 11$ and $4 \cdot 20$ respectively), no change in the position of the maxima being observed on the addition of fused sodium acetate (Jurd \& Horowitz, 1957); $\lambda_{\max .} 285,308$ and $360 \mathrm{~m} \mu$ (inflexion) in 2 mM-sodium ethoxide (log $\epsilon 4 \cdot 12,4 \cdot 05$ and $3 \cdot 79$ respectively); infrared spectrum 3340 (OH), 2900 (CH aromatic); 1652 (conjugated CO), 1603, 1575 (inflexion), 1512, 1452 (aromatic $\mathrm{C}-\mathrm{C}$ ) and $1435 \mathrm{~cm} .^{-1}$ (unknown) (Found: $\mathrm{C}, 58.6 ; \mathrm{H}$, $5 \cdot 3 ; 0 \cdot \mathrm{CH}_{3}, 19 \cdot 2$. $\mathrm{C}_{24} \mathrm{H}_{26} \mathrm{O}_{11}$ requires $\mathrm{C}, 58 \cdot 8 ; \mathrm{H}, 5 \cdot 3$; $0 . \mathrm{CH}_{3}, 19.0 \%$ ). The compound had $R_{F}$ values 0.04 (water), 0.15 [aq. $15 \%(\mathrm{v} / \mathrm{v})$ acetic acid], 0.59 [aq. $60 \%(\mathrm{v} / \mathrm{v})$ acetic acid], 0.51 [butan-1-ol-acetic acid-water $(20: 5: 11$, by vol.)] and 0.98 [aq. $75 \%(w / w)$ phenol].

Acetylation with acetic anhydride-pyridine under a wide variety of conditions failed to yield a crystalline product.

3',4',7-Tri-0-methyliso-orientin. The derivative was most satisfactorily prepared by methylation of iso-orientin with diazomethane as follows. A dry ethereal solution of diazomethane (about $100 \mathrm{ml}$.) generated from nitrosomethylurea $(3.5 \mathrm{~g}$.) was added to a solution of iso-orientin (130 mg.) in a mixture of methanol $(10 \mathrm{ml}$.) and acetone $\left(40 \mathrm{ml}\right.$.) at $5^{\circ}$. After $1 \mathrm{hr}$. the mixture was evaporated under vacuum to about $25 \mathrm{ml}$. and left overnight in a desiccator under slightly reduced pressure. The solid that separated was filtered, recrystallized to constant m.p. from ethanol and dried under reduced pressure to constant weight over $\mathrm{P}_{2} \mathrm{O}_{5}$ at $110^{\circ}$. The final yield was $38 \mathrm{mg}$. of cream-coloured needles, m.p. $233^{\circ}$, solidifying on further heating and remelting at $272^{\circ} ;[\alpha]_{D}^{25}-10.3 \pm 0.2^{\circ}$ (c 0.97 in pyridine); $\lambda_{\max .} 245,274$ and $344 \mathrm{~m} \mu$ in ethanol $(\log \epsilon 4 \cdot 16,4 \cdot 16$ and 4.28 respectively), no change in the position of the maxima being observed on the addition of fused sodium acetate (Jurd \& Horowitz, 1957); $\lambda_{\max .} 293$ and $313 \mathrm{~m} \mu$ in $2 \mathrm{~mm}$ sodium ethoxide ( $\log \epsilon 4.01$ and 4.00 respectively); infrared spectrum 3340 (OH), 2900 (CH aromatic), 1647 (conjugated $\mathrm{CO}) ; 1610,1570$ (inflexion), 1512, 1452 (aromatic C-C) and $1493 \mathrm{~cm} .^{-1}$ (unknown) (Found: $\mathrm{C}, 58.8 ; \mathrm{H}, 5.5$; $0 \cdot \mathrm{CH}_{3}, 19 \cdot 0$. $\mathrm{C}_{24} \mathrm{H}_{26} \mathrm{O}_{11}$ requires $\mathrm{C}, 58 \cdot 8 ; \mathrm{H}, 5 \cdot 3 ; \mathrm{O} \cdot \mathrm{CH}_{3}$, $19.0 \%$ ). The compound had $R_{F}$ values 0.28 (water), 0.52 [aq. $15 \%(v / v)$ acetic acid], 0.82 [aq. $60 \%(v / v)$ acetic acid], $0 \cdot 72$ [butan-1-ol-acetic acid-water $(20: 5: 11$, by vol.)] and 0.97 [aq. $75 \%(w / w)$ phenol].

Acetylation of the compound (28mg.) with acetic anhydride $\left(0.2 \mathrm{ml}\right.$.) and pyridine $\left(0.1 \mathrm{ml}\right.$.) at about $20^{\circ}$ for $12 \mathrm{hr}$. yielded a white crystalline acetate $(19 \mathrm{mg}$.$) , which$ after two recrystallizations from ethanol had m.p. $179^{\circ}$ (Found: $\mathrm{C}, 58 \cdot 1$; H, 5.0; $0 . \mathrm{CH}_{3}, 13 \cdot 7 ; \mathrm{CO} \cdot \mathrm{CH}_{3}, 29 \cdot 2$. $\mathrm{C}_{34} \mathrm{H}_{36} \mathrm{O}_{16}$ requires $\mathrm{C}, 58 \cdot 3 ; \mathrm{H}, 5 \cdot 1 ; 0 \cdot \mathrm{CH}_{3}, 13 \cdot 3 ; \mathrm{CO} \cdot \mathrm{CH}_{3}$, 30.7\%). 
Permanganate oxidation of orientin and iso-orientin trimethyl ethers. The compound (50 mg.) was suspended in water (3.0 ml.) and aq. $5 \%(\mathrm{w} / \mathrm{v}) \mathrm{NaOH}$ soln. $(0.5 \mathrm{ml}$.) was added. The mixture was heated to boiling point, removed from the flame and a solution of $\mathrm{KMnO}_{4}(0.2 \mathrm{~g}$.) in water $(2.0 \mathrm{ml}$.) was slowly added. The mixture was set aside for 10 min., ethanol (about 3 drops) was added and the $\mathrm{MnO}_{2}$ was removed by filtration. The filtrate was acidified with aq. $\mathrm{HCl}$ and extracted twice with ether $(5 \mathrm{ml}$.). Evaporation of the ether left a crystalline solid (about $6 \mathrm{mg}$.), which was purified by micro-sublimation under reduced pressure. The purified compound had m.p. $182^{\circ}$, unchanged by admixture with an authentic sample of veratric acid. In addition, the infrared spectra of the compound and of veratric acid were identical over the range $2-15 \mu$.

Interconversion of orientin and iso-orientin trimethyl ethers. The compound (5 mg.) in ethanol (1ml.) was refluxed with aq. $2 \mathrm{~N}-\mathrm{HCl}(2 \mathrm{ml}$.) for $\mathrm{lhr}$. The solution was cooled and extracted with pentan-1-ol, and the extract was examined by paper chromatography. In each case partial isomerization of the trimethyl ether was observed. The compound formed from $3^{\prime}, 4^{\prime}, 7$-tri- $O$-methylorientin was identical with $3^{\prime}, 4^{\prime}, 7$-tri- $O$-methyliso-orientin by chromatography in water, aq. $15 \%(v / v)$ acetic acid, aq. $60 \%(v / v)$ acetic acid, butan-1-ol-acetic acid-water $(20: 5: 11$, by vol.) and aq. $75 \%(w / w)$ phenol. Similarly, the compound formed from $3^{\prime}, 4^{\prime}, 7$-tri- $O$-methyliso-orientin was identical with $3^{\prime}, 4^{\prime}, 7$-tri- $O$-methylorientin in all these solvent systems. Under the above conditions interconversion of orientin and iso-orientin also took place but no interconversion of the $3^{\prime}, 4^{\prime}, 5,7$-tetra- $O$-methyl derivatives could be detected, even after more prolonged acid treatment.

\section{Nuclear-magnetic-resonance spectra of orientin, iso-orientin and various derivatives}

The n.m.r. spectra were recorded by Dr K. G. R. Pachler, Chemical Physics Group, C.S.I.R., Pretoria, on a Varian A-60 spectrometer with dimethyl sulphoxide as solvent and tetramethylsilane as internal standard $(\delta=0.00$ p.p.m. $)$.
The sample concentration was $30-100 \mathrm{mg}$. to $0.5 \mathrm{ml}$. of solution. Band positions are expressed as p.p.m. downfield with the standard as origin. The results are summarized in Table 1 and are compared with published data for related compounds.

\section{DISCUSSION}

The finding that the tetramethyl ethers of orientin and iso-orientin each consumed $2 \mathrm{~mol}$. of periodic acid/mol. was incompatible with the structures originally proposed for these compounds (Hörhammer, Wagner, Nieschlag \& Wildi, 1959). The results suggested further that orientin and isoorientin were isomeric and analyses of the parent compounds and various derivatives supported this view (Koeppen, 1962). The formation in each case of $1 \mathrm{~mol}$. of formic acid $/ \mathrm{mol}$. of tetramethyl ether on periodic acid oxidation of these compounds and the liberation in each case of glycerol on hydrolysis of the periodate-oxidized borohydride-reduced tetramethyl ethers thereupon established that both compounds possess hexopyranosyl residues (cf. Smith \& Van Cleve, 1955). The compatibility of these findings with the $C$-glycosyl nature of orientin and iso-orientin has been discussed (Koeppen, $1965 a$ ). The final identity of the hexopyranosyl residues of orientin and iso-orientin was established by the detection of $D$-glucose as a product of ferric chloride oxidation of the tetramethyl ethers, the co-occurrence of $\mathrm{D}$-arabinose being due to oxidative cleavage of the $\mathrm{C}_{(1)}-\mathrm{C}_{(2)}$ bond of the glycosyl residue. As D-arabinose has been isolated as the major water-soluble product of the ferric chloride oxidation of barbaloin (Hay \& Haynes, 1956) and as only traces of glucose could be detected on oxidation of this compound under the conditions

Table 1. $\delta$ values for orientin, iso-orientin, related compounds and various derivatives in dimethyl sulphoxide

* Doublet $\left(J_{6,8}=2.5 \mathrm{cyc}\right.$. $/ \mathrm{sec}$. $)$.

$\dagger$ Deuterochloroform used as solvent in these cases.

Orientin

Iso-orientin

Vitexin (Horowitz \& Gentili, 1964)

Vitexin (Batterham \& Highet, 1964)

Isovitexin (Horowitz \& Gentili, 1964)

Apigenin (Horowitz \& Gentili, 1964)

$3^{\prime}, 4^{\prime}, 7-$ Tri- $O$-methylorientin

$3^{\prime}, 4^{\prime}, 7-$ Tri-O-methyliso-orientin

4',7-Di-O-methylapigenin† (Lamberton, 1964)

$3^{\prime}, 4^{\prime}, 5,7-T e t r a-O$-methylorientin

$3^{\prime}, 4^{\prime}, 5,7-$ Tetra-O-methyliso-orientin

4',5,7-Tri-O-methylapigenin † (Horowitz \& Gentili, 1964)

$3^{\prime}, 4^{\prime}$, 5,7-Tetra-O-acetyl-luteolin $†$

\begin{tabular}{|c|c|c|c|}
\hline $5-\mathrm{OH}$ & 3-H & 6-H & $8-\mathrm{H}$ \\
\hline 13.20 & 6.67 & $6 \cdot 30$ & - \\
\hline \multirow[t]{2}{*}{$13 \cdot 62$} & $6 \cdot 70$ & - & 6.54 \\
\hline & $6 \cdot 77$ & 6.29 & - \\
\hline \multirow[t]{3}{*}{$13 \cdot 20$} & $6 \cdot 80$ & $6 \cdot 28$ & - \\
\hline & $6 \cdot 77$ & - & $6 \cdot 56$ \\
\hline & 6.76 & $6.24^{*}$ & $6.52^{*}$ \\
\hline $13 \cdot 29$ & 6.97 & $6 \cdot 49$ & - \\
\hline $13 \cdot 47$ & 6.97 & - & $6 \cdot 81$ \\
\hline 12.92 & 6.55 & $6 \cdot 36^{*}$ & $6 \cdot 46^{*}$ \\
\hline - & $6 \cdot 70$ & 6.57 & - \\
\hline- & $6 \cdot 78$ & - & $7 \cdot 12$ \\
\hline- & 6.50 & $6 \cdot 30^{*}$ & $6 \cdot 48^{*}$ \\
\hline- & 6.62 & $6.88^{*}$ & $7 \cdot 37 *$ \\
\hline
\end{tabular}


employed in these Laboratories, it would appear that the glycosyl-aglycone bond is more stable in barbaloin than in orientin or iso-orientin.

As orientin and iso-orientin have both been shown to contain luteolin nuclei (Hörhammer, Wagner \& Gloggengiesser, 1958; Seikel \& Bushnell, 1959; Koeppen et al. 1962) and as both contain glucopyranosyl residues, it seemed reasonable to expect that they might differ in the position of attachment of the glucosyl residue to the luteolin nucleus and that their interconvertibility might therefore be due to a Wessely-Moser rearrangement of the flavone nucleus. Such a rearrangement would require the presence of a free 5-hydroxyl group, and the possibility of such a mechanism being operative for the orientin-iso-orientin interconversion is supported by the finding that the $3^{\prime}, 4^{\prime}, 5,7$-tetramethyl ethers are not interconvertible whereas the $3^{\prime}, 4^{\prime}, 7$-trimethyl ethers are. That the tri- $O$. methyl derivatives prepared are indeed the $3^{\prime}, 4^{\prime}, 7$ trimethyl ethers is confirmed by the following considerations. (1) Permanganate oxidation of the trimethyl ethers yields veratric acid in each case. Thus the $3^{\prime}$ - and 4' -hydroxyl groups are methylated. (2) The positions of the ultraviolet-absorption maxima remain unaltered on the addition of fused sodium acetate to ethanolic solutions of the trimethyl ethers. This indicates blockage of the 7-hydroxyl group in each compound (Jurd \& Horowitz, 1957). In addition, the spectra of the compounds in sodium ethoxide solution are typical of flavones whose only free hydroxyl group is in the 5-position (Jurd, 1962). (3) The strongly positive brown ferric chloride reactions of the trimethyl ethers indicates the presence of a free 5-hydroxyl group in each case, and the ability of the compounds to form aluminium chelates that fluoresce brightly under ultraviolet light indicates the presence of a peri-hydroxycarbonyl grouping in each compound (cf. Koeppen, 1965b). (4) The n.m.r. spectra show a signal at $\delta=13 \cdot 2-13.5$ p.p.m. due to a strongly hydrogen-bonded hydroxyl proton in each compound.

The n.m.r. spectra of orientin, iso-orientin and various derivatives (Table 1) support the identification of these compounds as ring-isomeric $C$ glycosylflavones.

As with vitexin and isovitexin (Horowitz \& Gentili, 1964), only one A-ring proton was observed in the spectra of orientin and iso-orientin. In each case this appeared as a simple unsplit signal, indicating the absence of a coupled meta-proton. By contrast, the coupled 6- and 8-protons of luteolin tetra-acetate and of apigenin and its methyl ethers appeared as doublets with $J_{6,8}=2 \cdot 5 \mathrm{cyc}$./sec as expected. These observations support the chemical evidence that the positions meta to the A-ring protons in orientin and in iso-orientin are substituted.
Although chemical methods have established unequivocally that the glycosyl residue of vitexin is attached to the 8-position of the flavone nucleus (Evans, McGookin, Jurd, Robertson \& Williamson, 1957), all attempts to apply these methods to the determination of the position of the glycosyl residues in orientin and in iso-orientin have so far been without success. However, the solubility and chromatographic properties of orientin strongly suggested that it was more closely related to vitexin than was iso-orientin, and it was accordingly proposed (Koeppen, 1964) that orientin was the 8and iso-orientin the 6-substituted flavone. The n.m.r. data presented in Table 1 support this view. Thus the spectrum of orientin corresponds closely to that of vitexin, and, similarly, the spectra of isovitexin and iso-orientin are in close agreement, particularly with regard to the resonance position of the A-ring benzenoid proton in each case. In compounds such as apigenin and luteolin tetraacetate, which possess protons in both the 6- and the 8-positions, the upfield doublet of the characteristic two-doublet pattern obtained has been assigned to the 6-proton (Massicot \& Marthe, 1962). This is supported by comparison of the spectra of vitexin and isovitexin with these compounds, the 6-proton of vitexin appearing at higher field than the 8-proton of iso-vitexin. Similarly, the chemical and physical properties of a compound isolated from Eucalyptus waxes by Lamberton (1964) indicated that the compound was 5-hydroxy-4',7-dimethoxy6-methylflavone and not the 8-C-methyl analogue. Thus a signal at $\delta=6 \cdot 46$ p.p.m. in the n.m.r. spectrum of this compound in deuterochloroform was ascribed to the 8-proton and this was confirmed by the appearance of an additional signal at higher field $(\delta=6 \cdot 37$ p.p.m. $)$ in a Wessely-Moser rearrangement mixture containing the 6- and 8-Cmethylflavone. In orientin, the benzenoid A-ring proton appears upfield from that in iso-orientin, and the same relative positions of the protons are observed when comparing $3^{\prime}, 4^{\prime}, 7$-tri-O-methylorientin with $3^{\prime}, 4^{\prime}, 7$-tri- $O$-methyliso-orientin and $3^{\prime}, 4^{\prime}, 5,7$-tetra- $O$-methylorientin with $3^{\prime}, 4^{\prime}, 5,7$-tetra$O$-methyliso-orientin. Thus comparison of the n.m.r. spectra of the parent compounds and their tri- and tetra-O-methyl derivatives all indicate that orientin is the 8-and iso-orientin the 6-substituted flavone. Further evidence to this effect is provided by comparison of the position of the 5-hydroxyl proton resonance in orientin, iso-orientin and their $3^{\prime}, 4^{\prime}, 7$-tri-O-methyl derivatives. Thus Batterham \& Highet (1964) observed that the low-field resonance of the strongly hydrogen-bonded 5hydroxyl proton in flavones was shifted to even lower field by substitution of a glycosyl residue in the para-position as in vitexin. It might therefore be expected that substitution of such a residue in the 
<smiles></smiles>

(I)

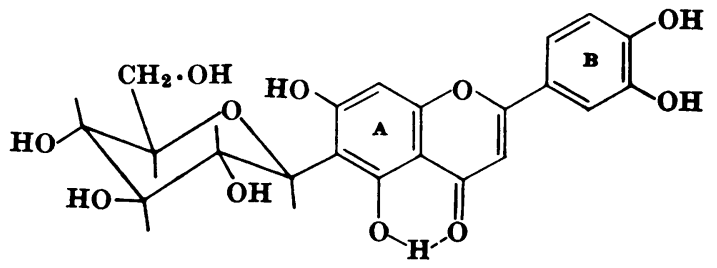

(II)

ortho-position might result in even greater unshielding of the 5-hydroxyl proton and this indeed appears to be the case. Thus the 5-hydroxyl proton of orientin is observed at $\delta=13 \cdot 20$ p.p.m., the same position as in vitexin, whereas in iso-orientin it appears further downfield ( $\delta=13.62$ p.p.m.). Similarly, in $3^{\prime}, 4^{\prime}, 7$-tri- $O$-methyliso-orientin this signal appears at lower field than in $3^{\prime}, 4^{\prime}, 7$-tri- $O$-methylorientin.

The benzylic proton referred to by Horowitz \& Gentili (1964) appears in the same position for orientin $(\delta=4.75$ p.p.m. $)$ and iso-orientin $(\delta=$ 4.67 p.p.m.) as for vitexin and iso-vitexin respectively. In each case it is recognizable as a doublet
( $J=10 \cdot 0$ cyc./sec.), thereby indicating that orientin and iso-orientin, like vitexin, possess $\beta$ - $C$-glucopyranosyl residues.

By chemical and spectral evidence orientin is therefore formulated as 8-C- $\beta$-D-glucopyranosyl$3^{\prime}, 4^{\prime}, 5,7$-tetrahydroxyflavone (I) and iso-orientin as the 6-substituted luteolin analogue (II).

The authors are grateful to Mr P. de K. du Preez for technical assistance.

\section{REFERENCES}

Batterham, T. J. \& Highet, R. J. (1964). Aust. J. Chem. $17,428$.

Dyer, J. R. (1956). Meth. biochem. Anal. 3, 111.

Evans, W. H., McGookin, A., Jurd, L., Robertson, A. \& Williamson, W. R. N. (1957). J. chem. Soc. p. 3510.

Hay, J. E. \& Haynes, L. J. (1956). J. chem. Soc. p. 3141.

Hörhammer, L., Wagner, H. \& Gloggengiesser, F. (1958). Arch. Pharm., Berl., 291, 126.

Hörhammer, L., Wagner, H., Nieschlag, H. \& Wildi, G. (1959). Arch. Pharm., Berl., 292, 380.

Horowitz, R. M. \& Gentili, B. (1964). Chem. \& Ind. p. 498. Jurd, L. (1962). In The Chemistry of Flavonoid Compounds, p. 107. Ed. by Geissman, T. A. Oxford: Pergamon Press Ltd.

Jurd, L. \& Horowitz, R. M. (1957). J. org. Chem. 22, 1618. Koeppen, B. H. (1962). Chem. \& Ind. p. 2145.

Koeppen, B. H. (1964). Z. Naturf. 19 b, 173.

Koeppen, B. H. (1965a). Phytochemistry 4, 639.

Koeppen, B. H. (1965b). J. Chromat. 18, 604.

Koeppen, B. H., Smit, C. J. B. \& Roux, D. G. (1962). Biochem.J. 83, 507.

Lamberton, J. A. (1964). Aust. J. Chem. 17, 692.

Massicot, J. \& Marthe, J.-P. (1962). Bull. Soc. chim. Fr. p. 1962.

Roux, D. G. \& Maihs, A. E. (1960). Biochem. J. 74, 44.

Seikel, M. K. \& Bushnell, A. J. (1959). J. org. Chem. 24, 1995.

Smith, F. \& Van Cleve, J. W. (1955). J. Amer. chem. Soc. 77, 3091. 